\title{
Magnetic x-ray standing waves
}

\section{Kamiński, H. Schulz-Ritter, M. Tolkiehn}

Deutsches Elektronen-Synchrotron (DESY), Photon Science, Notkestraße 85, 22607 Hamburg, Germany, michal.kaminski@desy.de

Magnetic x-ray standing waves (MXSW) - a combination of x-ray standing waves (XSW) [1] and x-ray magnetic circular dichroism (XMCD) - is a new method for direct investigation of magnetic structure of crystals and thin films on the atomic level. In the regular XSW technique a standing wave emerging in the region where incoming and Bragg reflected waves interfere is employed to study atomic positions in element specific manner. The standing wave has a periodicity of the lattice and moves by half of its period as the sample is rocked through the reflection domain. This movement across the lattice causes modulations in the amount of emitted fluorescence - their character is characteristic for a distribution of given atomic kind. This gives - in contrary to diffraction methods - a direct, element specific structural information. In MXSW, additional magnetic sensitivity is achieved by using circularly polarised incoming wave and magnetising the sample. The normalised difference between fluorescence yields recorded for each helicity/magnetic field orientation (XMCD signal) is proportional to the distribution of magnetic atoms and their magnetic moments. This makes MXSW site, element and magnetic sensitive method.

The theoretical framework of MXSW method is based on dynamical theory of x-ray diffraction and time-dependent perturbation theory. The first is used to describe the phenomena of the scattering of x-rays by the crystal lattice and yields a form of the wavefield inside the crystal for circularly polarised incident wave. The latter provides a tool to evaluate the absorption cross-section for the considered wavefield. What is obtained finally is an angular dependence of XMCD signal, which similarly as a single fluorescence yield in XSW method, exhibits variations dependent on the distribution of magnetic atoms.

The Fig. 1 shows an exemplary, simulated MXSW signal for the magnetite crystal, (004) reflection. The insets present schematically the magnetic structure and the positions of the standing wave at the low (marked by green colour) and high (blue) angular side of the reflection domain. Iron ions in the magnetite structure are arranged in two sublattices - octahedrally (marked by blue colour) and tetrahedrally (green) coordinated ones. Since the magnetic moment on each of two sublattices is different and oriented opposite, the contribution of the sublattices to the overall XMCD signal changes depending on the position of the nodes and antinodes of the standing wave. The variations are characteristic for this arrangement of the iron atoms and their shape would be different for any other one. Therefore, the MXSW signal directly provides information about the magnetic structure.

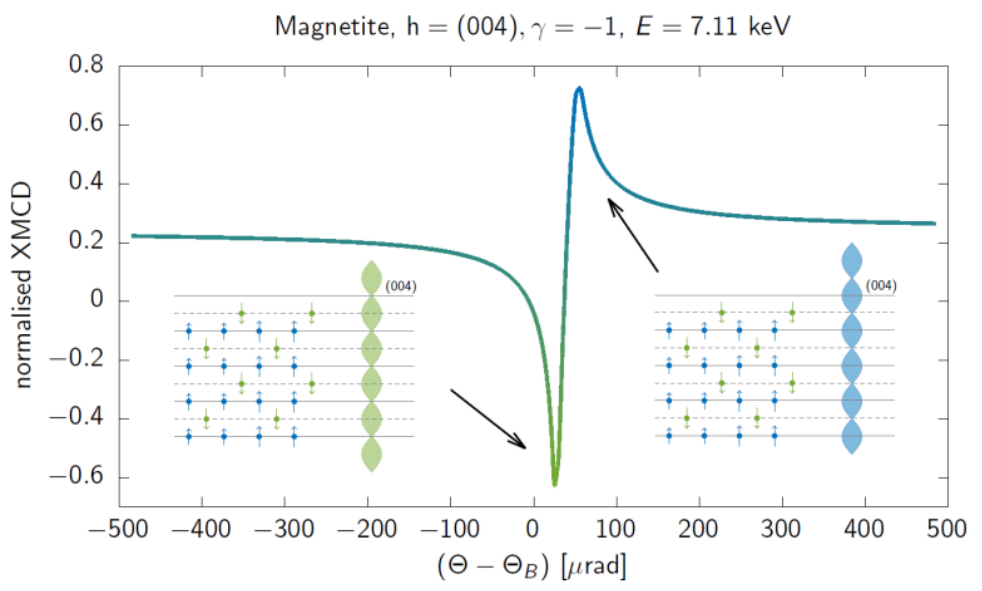

Figure 1. MXSW signal for magnetite, (004) reflection. The schemas show a unit cell of magnetite and standing waves formed on the low (green) and high (blue) angular side of the reflection domain.

The first experiment aiming at proving the feasibility of the method and confirming the established theory was performed at PETRA III synchrotron on the single crystal sample of $\mathrm{Pt}_{3} \mathrm{Co}$ alloy. The measurements were conducted at the $\mathrm{Pt}_{3}$ absorption edge. A clear variation in XMCD signal of the magnetic origin was observed. As a next step, an experiment on magnetite is planned to show the power of the method to probe the arrangement of the magnetic ions.

[1] Zegenhagen, J., Kazimirov, A., The X-Ray standing Wave Technique: Principles and Applications (2013).

Keywords: x-ray standing waves; magnetic structure; direct method 